\title{
Multiparametric cardiovascular magnetic resonance surveillance of acute cardiac allograft rejection and characterisation of transplantation-associated myocardial injury: a pilot study
}

Christopher A Miller ${ }^{1,2,3^{*}}$, Josephine H Naish², Steven M Shaw ${ }^{1,3}$, Nizar Yonan ${ }^{1,3}$, Simon G Williams ${ }^{1,3}$, David Clark ${ }^{4}$, Paul W Bishop ${ }^{5}$, Mark P Ainslie ${ }^{1,2}$, Alex Borg ${ }^{1}$, Glyn Coutts ${ }^{6}$, Geoffrey JM Parker ${ }^{2}$, Simon G Ray ${ }^{1,3}$ and Matthias Schmitt ${ }^{1,2}$

\begin{abstract}
Background: Serial surveillance endomyocardial biopsies are performed in patients who have recently undergone heart transplantation in order to detect acute cardiac allograft rejection (ACAR) before symptoms occur, however the biopsy process is associated with a number of limitations. This study aimed to prospectively and longitudinally evaluate the performance of multiparametric cardiovascular magnetic resonance (CMR) for detecting and monitoring ACAR in the early phase post-transplant, and characterize graft recovery following transplantation.

Methods: All patients receiving a heart transplant at a single UK centre over a period of 25 months were approached within one month of transplantation. Multiparametric CMR was prospectively performed on the same day as biopsy on four separate occasions ( 6 weeks, 10 weeks, 15 weeks and 20 weeks post-transplant). CMR included assessment of global and regional ventricular function, myocardial tissue characterization (T1 mapping, T2 mapping, extracellular volume, LGE) and pixel-wise absolute myocardial blood flow quantification. CMR parameters were compared with biopsy findings. As is standard, grade 2R or higher ACAR was considered significant.

Results: 88 CMR-matched biopsies were performed in 22 patients. Eight (9\%) biopsies in 5 patients demonstrated significant ACAR. Significant ACAR was associated with a reduction in circumferential strain $(-12.7 \pm 2.5 \%$ vs. $-13.7 \pm$ $3.6 \%, p=0.047)$ but there was considerable overlap between groups. Whilst trends were observed between ACAR and proposed CMR markers of oedema, particularly after adjusting for primary graft dysfunction, differences were not significant. Significant improvements were seen in markers of graft structure and contractility, oedema and microvascular function over the period studied, although few parameters normalised.
\end{abstract}

Conclusions: This study provides novel insight into the myocardial injury associated with transplantation, and its recovery, however multiparametric CMR was not able to accurately detect ACAR during the early phase post-transplantation.

Keywords: Cardiovascular magnetic resonance, Heart transplantation, Acute rejection

\footnotetext{
*Correspondence: chrismiller@doctors.org.uk

${ }^{1}$ North West Heart Centre and The Transplant Centre, University Hospital of

South Manchester, Manchester, UK

${ }^{2}$ Centre for Imaging Sciences \& Biomedical Imaging Institute, University of

Manchester, Manchester, UK

Full list of author information is available at the end of the article
} 


\section{Background}

Acute cardiac allograft rejection (ACAR) affects approximately $20 \%$ of patients in the first year post-transplantation, and represents a leading cause of death during this period [1]. Moreover, even when apparently successfully treated, an episode of ACAR occurring during the first year confers higher two- and four-year mortality in patients surviving beyond the first year [1].

Clinical features of ACAR are unreliable, with patients usually remaining asymptomatic until hemodynamic complications ensure. Routine screening is therefore performed in order to detect ACAR, and hence augment immunosuppressive therapy, at an earlier stage, with the aim of preventing progression to more severe disease, and potentially reducing the risk of long-term complications. ACAR surveillance is performed via histological analysis of right ventricular myocardial tissue obtained at endomyocardial biopsy, and patients undergo frequent biopsies (10-15) during the first post-operative year. However, the procedure is invasive (complication rate $0.5-1.5 \%$ ), expensive and disliked by patients, factors which prevent more frequent monitoring, limiting optimal titration of immunosuppressive therapy [2]. Furthermore the sensitivity of the technique is limited by sampling error due to the patchy nature of ACAR and variability in interpretation of the histological appearances [3].

Cardiovascular magnetic resonance (CMR) is a potentially attractive screening modality for ACAR due to its lack of ionizing radiation and its multiparametric nature, i.e. its ability to assess multiple aspects of myocardial injury in a single examination, including global and regional ventricular function, myocardial oedema (quantitative T1 and T2 mapping), myocardial blood flow and focal (late gadolinium enhancement, LGE) and diffuse (extracellular volume; ECV) myocardial interstitial expansion.

Systematic evaluation of multiparametric CMR for diagnosing ACAR has not been reported to date. The aims of this prospective study were to assess the performance of multiparametric CMR for detecting and monitoring ACAR, to characterize the graft injury following transplantation and to longitudinally evaluate graft recovery in the early phase post-transplantation.

\section{Methods}

\section{Patients and study design}

All patients receiving a heart transplant at a single center over a 25 month period were prospectively approached within one month of undergoing transplantation (Figure 1). The only exclusion criteria were contraindications to CMR scanning and inability to give informed consent. The work was conducted in accordance with the Declaration of Helsinki, an ethics committee of the UK National Research Ethics Service approved the study

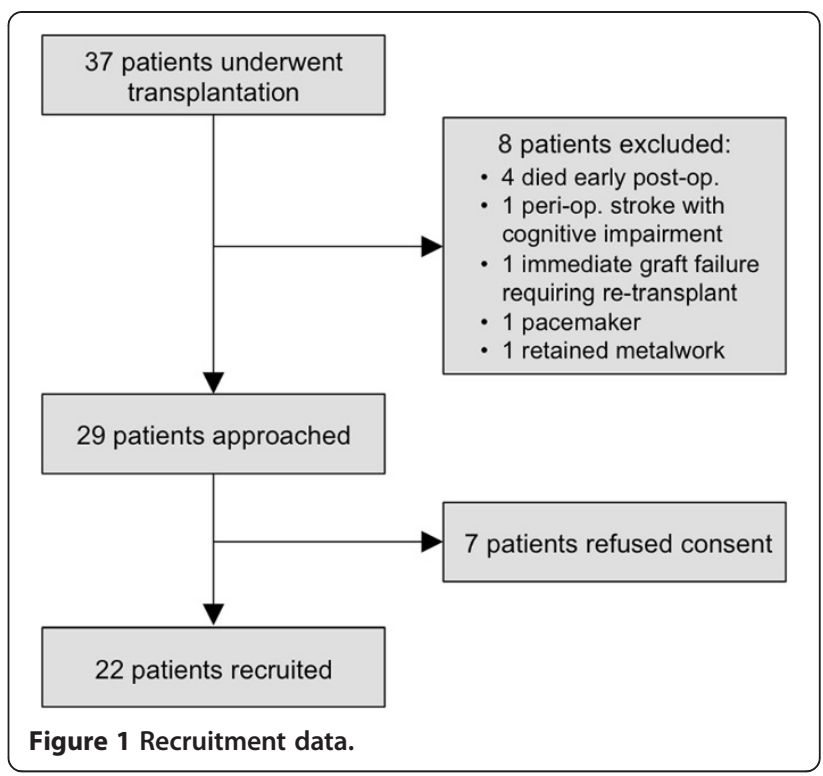

$(09 / \mathrm{H} 1003 / 100)$ and written informed consent was obtained from all participants.

Each patient was prospectively scanned on the same day as biopsy on four separate occasions. As pre-specified, scans were performed to coincide with biopsies scheduled for 6 weeks, 10 weeks, 15 weeks and 20 weeks posttransplant. Investigators were blinded to biopsy results i.e. scans were not performed on the basis of confirmed or suspected ACAR. CMR was performed immediately before or after biopsy, the order of which was determined randomly. Due to concerns over cumulative gadolinium contrast agent dose, the parts of the protocol requiring contrast agent were performed in two out of the four scans in each patient. Patients with an estimated glomerular filtration rate of $35 \mathrm{ml} / \mathrm{min} / 1.73 \mathrm{~m}^{2}$ or less did not undergo the parts of the protocol requiring contrast agent. In addition, 10 age- and sex-matched healthy volunteers (asymptomatic, no risk factors, normal physical examination, normal electrocardiogram) were recruited to undergo CMR scanning.

\section{CMR acquisition}

CMR was performed at 1.5T (Avanto; Siemens Medical Imaging, Germany) with a 32-element phased-array coil. Steady-state free precession cine images were acquired in standard long-axis views and in a stack of short-axis slices covering the left ventricle (LV). Short-axis tagged images were acquired at basal, mid and apical ventricular levels using a segmented k-space fast gradient echo sequence with spatial modulation of magnetization in orthogonal planes. A single-shot modified Look Locker inversion recovery (MOLLI) sequence was acquired in short-axis at mid-ventricular level before contrast agent was administered, and 15-minutes after the contrast 
agent 'top-up' (see below) [4]. Same day haematocrit was measured. A $\mathrm{T}_{2}$-prepared SSFP sequence was acquired in short-axis at mid-ventricular level in late diastole at preparation times, $\mathrm{T} 2_{\text {prep }}$, of 0, 24, 45, $65 \mathrm{~ms}$. [5] Perfusion imaging was performed as described previously [6]. Briefly, a saturation recovery gradient echo sequence was acquired during adenosine stress and at rest following a $0.05 \mathrm{mmol} / \mathrm{kg}$ bolus of gadolinium-based contrast agent (gadopentetate dimeglumine; Gd-DTPA; Magnevist; Bayer Healthcare, Germany). Following rest perfusion image acquisition, a further $0.1 \mathrm{mmol} / \mathrm{kg}$ of contrast agent was administered ('top-up') to bring the total dose to $0.2 \mathrm{mmol} /$ $\mathrm{kg}$. Standard late gadolinium enhancement (LGE) imaging was performed at least 10 minutes following the contrast agent 'top-up'.

\section{CMR analysis}

LV mass, end-diastolic volume, end-systolic volume and ejection fraction $(\mathrm{EF})$ were quantified from steady-state free precession images using CMRtools (Cardiovascular Imaging Solutions, UK) [7]. Peak systolic circumferential strain $(\varepsilon c c)$ and strain rate (systolic and early-diastolic) were measured from mid-ventricular short-axis tagged images using SinMod (inTag, CREATIS lab, France, and Maastricht University, The Netherlands, v5.0) [8]. Basal and apical short-axis rotation, calculated from tagged images using the same software, and epicardial areas, were incorporated into a custom-written algorithm (Microsoft Excel using Visual Basic) in order to calculate twist (basal rotation-apical rotation), normalized twist (twist angle divided by distance between slices) and torsion (circumferential-longitudinal shear angle; calculated by multiplying normalized twist by mean of basal and apical epicardial radii).

Myocardial $\mathrm{T}_{1}$ relaxation time was measured as described previously [9]. Briefly, voxel-wise $T_{1}$ relaxation maps ('T1 mapping') were obtained from the MOLLI images using a 3 parameter fit to signal intensity, $\mathrm{S}$ as a function of effective inversion time (TIeff) according to $\mathrm{S}(\mathrm{TI}$ eff $)=\mathrm{A}-\mathrm{Be}^{\left(-\mathrm{TIeff} / \mathrm{T} 1^{*}\right)}$ and $\mathrm{T}_{1}$ was calculated as $\mathrm{T}_{1}=$ $\mathrm{T}_{1} *((\mathrm{~B} / \mathrm{A})-1)$. After applying a heart-rate correction algorithm, mean mid-ventricular pixel $\mathrm{T}_{1}$ relaxation times before and after contrast were then used to calculate myocardial extracellular volume (ECV) according the following formula: $E C V=\lambda \times(1-$ haematocrit $)$. Where the partition coefficient, $\lambda=\Delta R_{1}$ (myocardium) $/ \Delta R_{1}$ (blood). $\Delta \mathrm{R}_{1}$ is proportional to contrast agent concentration. $\Delta \mathrm{R}_{1}=\mathrm{R}_{1}$ (post-contrast) $-\mathrm{R}_{1}$ (pre-contrast).

Voxel-wise $\mathrm{T}_{2}$ relaxation maps ('T2 mapping') were obtained from the T2-prepared SSFP images using a linear least squares fit to the log transformed signal intensity in each voxel according to $\operatorname{In}(S)=\operatorname{In}\left(S_{0}\right)-$ $\left(\mathrm{T} 2_{\text {prep }} / \mathrm{T}_{2}\right)$ where $\mathrm{S}$ is the measured signal intensity and $\mathrm{S}_{0}$ is the fitted signal intensity corresponding to no $\mathrm{T}_{2}$-preparation $\left(T 2_{\text {prep }}=0\right)$.
Perfusion quantification was performed as described previously [6]. Briefly, signal intensity curves were extracted from the average signal in the blood pool, to provide an arterial input function, and on a voxel-wise basis from myocardial regions of interest. Signal intensity was converted to contrast agent concentration [10]. Perfusion values were obtained on a voxel-wise basis using generalized Tikhonov deconvolution with a b-spline representation of the impulse response function [11]. Myocardial perfusion reserve (MPR) was calculated by dividing median hyperaemic myocardial blood flow (MBF) by median resting MBF. LGE images were reported visually by 2 experienced operators and the presence or absence of LGE, and its distribution pattern, were recorded.

\section{Endomyocardial biopsy and histological analysis}

Right ventricular biopsy was performed in a standard manner. Five to ten tissue samples were obtained from the right ventricular septum, stained with haematoxylin and eosin and analysed with light microscopy. Tissue was graded according to the 2005 Revision of the International Society for Heart and Lung Transplantation (ISHLT) Standardized Cardiac Biopsy Grading Criteria [12]. Immunosuppressive therapy is generally augmented at grade $2 \mathrm{R}$ or higher and thus grades $2 R-3 R$ were considered 'significant' ACAR and grades $0 R-1 R$ 'non-significant' ACAR. As is the clinical policy at our Institution, immunopathological assessment was performed if histological features of antibody-mediated rejection were present or if there was a high clinical suspicion of ACAR in the absence of significant acute cellular rejection. Patients treated for ACAR on the basis of high clinical suspicion alone (i.e. in the absence of $\geq$ grade $2 R$ acute cellular rejection or antibody mediated rejection) were also recorded. Patients were followed up in order to determine ACAR grade on the biopsy subsequent to those included in study.

\section{Statistical analysis}

All data was analysed in a blinded fashion, with independent analysis of CMR and biopsy data. Statistical analysis was performed using SPSS (IBM, USA; v20). Continuous variables are expressed as mean $\pm S D$ unless stated. An independent-samples $t$ test (or Mann-Whitney $U$ test where appropriate) was used to compare baseline transplant patient and healthy volunteer demographic data. CMR data were assessed according to ACAR grade using generalized estimating equations (GEE) with an ordinal logistic model in order to adjust for the repeated measurements over time within in each subject. Subsequently, CMR data were dichotomized according to the presence ( $\geq$ grade $2 \mathrm{R}$ ) or absence (grade $0 \mathrm{R}-1 \mathrm{R}$ ) of significant ACAR and compared using GEE with a binary logistic model. CMR data from healthy volunteers and transplant patients without significant ACAR were compared using 
the same method. A GEE linear regression model was used to compare CMR data between scans performed at different time points, and also to assess the relationship between CMR parameters. CMR data from transplant patients at a single time-point were compared with data from healthy volunteers using an independent $t$ test.

\section{Results}

\section{Study population and biopsy results}

Twenty-two patients were recruited (Figure 1). Demographic data are presented in Table 1. Each patient underwent CMR on the same day as biopsy on four separate occasions i.e. 88 biopsies and corresponding CMR scans in total. Median (IQR) timing of the four scans was 6.9 (4.9-8.3) weeks, 10.9 (9.5-12.6) weeks, 16.6 (13.2-19.6) weeks and 22.3 (19.6-26.3) weeks post-transplantation. Five patients (23\%) displayed grade 2R ACAR on biopsies included in the study. Three of these patients demonstrated grade $2 \mathrm{R}$ ACAR on 2 separate occasions. Of the 88 biopsies included in the study, 48 (55\%) were grade 0R, $29(33 \%)$ were grade $1 \mathrm{R}, 8(9 \%)$ were grade $2 \mathrm{R}, 0$ were grade $3 \mathrm{R}$ and $3(3 \%)$ were inadequate (insufficient tissue to allow accurate interpretation). No biopsy demonstrated antibody mediated rejection and no patient was treated for ACAR on the basis of high clinical suspicion alone. Eight (36\%) patients underwent CMR without contrast agent (5 patients had severe renal dysfunction and 3 patients refused consent). Healthy volunteers and transplant recipients were well matched (Table 1 ).

\section{ACAR}

Multiparametric CMR data is presented in Table 2. Whilst $\varepsilon c c$, native $T_{1}$ and $T_{2}$ data demonstrated a trend towards a deterioration as ACAR severity increased, only ecc showed a significant difference between significant and nonsignificant ACAR, although the absolute difference was small and there was considerable overlap between groups (Table 2, Figures 2 and 3). The outcome was no different if analysis was performed using CMR data from septal segments only. Pericardial effusion, seen on 9 scans (10\%), was unrelated to ACAR ( $\mathrm{p}=0.864)$. Only one biopsy demonstrating significant ACAR was accompanied by a CMR scan that was performed with contrast agent, hence meaningful assessment of perfusion, LGE and ECV data with regards to ACAR was not possible.

Six of the 8 subsequent biopsies to those showing significant ACAR demonstrated non-significant ACAR after immunosuppressive treatment and had corresponding CMR data (one patient demonstrated grade $2 \mathrm{R}$ on 2 consecutive biopsies and one patient showed grade $2 \mathrm{R}$ ACAR on biopsy number 4 hence no follow-up CMR data was available). On these 'convalescent' scans, CMR parameters were seen to return to 'baseline' levels $(\varepsilon c c:-15.0 \pm 2.5 \%$, native $\mathrm{T}_{1}: 1048 \pm 36 \mathrm{~ms}, \mathrm{~T}_{2}: 55.6 \pm 3.4 \mathrm{~ms}$ ).
Table 1 Baseline subject characteristics

\begin{tabular}{|c|c|c|c|}
\hline & $\begin{array}{l}\text { Transplant } \\
\text { patients } \\
(\mathrm{n}=22)\end{array}$ & $\begin{array}{l}\text { Healthy } \\
\text { volunteers } \\
(n=10)\end{array}$ & $\mathrm{p}$ value \\
\hline Male & 17 (77\%) & $7(70 \%)$ & 0.660 \\
\hline Age (years) & $49 \pm 10$ & $49 \pm 8$ & 0.961 \\
\hline Non-white & $2(9 \%)$ & $1(10 \%)$ & 0.676 \\
\hline $\mathrm{BSA}\left(\mathrm{m}^{2}\right)$ & $1.86 \pm 0.20$ & $1.94 \pm 0.19$ & 0.290 \\
\hline $\mathrm{BMI}\left(\mathrm{kg} / \mathrm{m}^{2}\right)$ & $24.7 \pm 3.6$ & $25.7 \pm 4.1$ & 0.484 \\
\hline $\mathrm{eGFR}\left(\mathrm{mL} / \mathrm{min} / \mathrm{m}^{2}\right)$ & $61 \pm 24$ & $89 \pm 13$ & $<0.001$ \\
\hline $\mathrm{HR}(\mathrm{bpm})$ & $89 \pm 15$ & $60 \pm 6$ & $<0.001$ \\
\hline Systolic BP (mmHg) & $135 \pm 17$ & $111 \pm 8$ & $<0.001$ \\
\hline \multicolumn{4}{|l|}{ Indication for transplant } \\
\hline DCM & $12(55 \%)$ & - & \\
\hline $\mathrm{IHD}$ & $6(27 \%)$ & - & \\
\hline ARVC & $2(9 \%)$ & - & \\
\hline $\mathrm{HCM}$ & $1(5 \%)$ & - & \\
\hline Re-transplant & $1(5 \%)$ & - & \\
\hline Pregnancy prior to transplantation & $3(14 \%)$ & - & \\
\hline VAD prior to transplantation & $1(5 \%)$ & - & \\
\hline Donor age (years) & $42 \pm 7$ & - & \\
\hline Donor male & 17 (77\%) & - & \\
\hline Donor: recipient gender match & $18(82 \%)$ & - & \\
\hline \multicolumn{4}{|l|}{ Donor cause of death } \\
\hline $\mathrm{ICH}$ & $13(59 \%)$ & - & \\
\hline Head trauma & $5(23 \%)$ & - & \\
\hline Other & $4(18 \%)$ & - & \\
\hline CMV donor pos., recipient neg. & $5(23 \%)$ & - & \\
\hline Ischaemic time (min) & $184 \pm 52$ & - & \\
\hline \multicolumn{4}{|l|}{ Induction immunosuppression } \\
\hline rATG & $21(95 \%)$ & - & \\
\hline Basiliximab & $1(5 \%)$ & - & \\
\hline \multicolumn{4}{|l|}{ Initial immunosuppression regime } \\
\hline Cyclosporine/prednisolone/MMF & $21(95 \%)$ & - & \\
\hline Tacrolimus/prednisolone/MMF & $1(5 \%)$ & - & \\
\hline ICU stay (days) & $17 \pm 19$ & - & \\
\hline Hospital stay (days) & $36 \pm 23$ & - & \\
\hline
\end{tabular}

BSA indicates body surface area; BMI body mass index; eGFR estimated glomerular filtration rate; HR heart rate; BP blood pressure; DCM indicates dilated cardiomyopathy; IHD ischemic heart disease; ARVC arrhythmogenic right ventricular cardiomyopathy; HCM hypertrophic cardiomyopathy; (Re-transplant: this patient suffered immediate graft failure and therefore underwent a second transplantation); VAD ventricular assist device; ICH spontaneous intracranial haemorrhage; CMV cytomegalovirus; rATG rabbit antithymocyte globulin; MMF Mycophenolate mofetil; ICU intensive care unit.

Five biopsies demonstrating non-significant ACAR were followed by a subsequent biopsy performed within 28 days that demonstrated grade $2 \mathrm{R}$. CMR data corresponding to these biopsies demonstrated $\varepsilon \mathrm{cc}(-13.5 \pm 2.2 \%)$, native $\mathrm{T}_{1}$ $(1090 \pm 56 \mathrm{~ms})$ and $\mathrm{T}_{2}(58.8 \pm 3.1 \mathrm{~ms})$ values that were 
Table 2 Cardiovascular magnetic resonance findings according to the presence (grade 2R) or absence (grade 0R-1R) of significant acute cardiac allograft rejection

\begin{tabular}{|c|c|c|c|c|}
\hline & Healthy volunteers & No rejection (OR - 1R) & Rejection (2R) & $p$ value \\
\hline LVEDVI $\left(\mathrm{mL} / \mathrm{m}^{2}\right)$ & $85.9 \pm 8.1$ & $84.2 \pm 21.7$ & $73.6 \pm 15.7$ & 0.287 \\
\hline LVESVI $\left(\mathrm{mL} / \mathrm{m}^{2}\right)$ & $28.5 \pm 4.3$ & $35.5 \pm 21.9$ & $28.1 \pm 10.9$ & 0.329 \\
\hline LVEF (\%) & $66.9 \pm 4.2^{*}$ & $60.4 \pm 13.7$ & $62.2 \pm 9.3$ & 0.655 \\
\hline LVMI $\left(\mathrm{g} / \mathrm{m}^{2}\right)$ & $46.4 \pm 7.3^{\dagger}$ & $62.2 \pm 58.4$ & $58.4 \pm 7.3$ & 0.362 \\
\hline$\varepsilon \subset c(\%)$ & $-20.7 \pm 1.0^{\dagger}$ & $-13.7 \pm 3.6$ & $-12.7 \pm 2.5$ & 0.047 \\
\hline Peak systolic SR (1/s) & $-1.17 \pm 0.14$ & $-1.05 \pm 0.31$ & $-1.06 \pm 0.26$ & 0.568 \\
\hline Early diastolic SR (1/s) & $0.37 \pm 0.20^{*}$ & $0.25 \pm 0.13$ & $0.26 \pm 0.13$ & 0.917 \\
\hline Normalised twist $(\% / \mathrm{mm})$ & $0.34 \pm 0.12^{*}$ & $0.26 \pm 0.10$ & $0.29 \pm 0.08$ & 0.151 \\
\hline Torsion (mm) & $8.99 \pm 3.11$ & $7.49 \pm 2.86$ & $8.11 \pm 1.93$ & 0.237 \\
\hline Time to peak torsion (\%AVC) & $96.4 \pm 5.5$ & $97.7 \pm 8.6$ & $100.6 \pm 8.0$ & 0.555 \\
\hline Native $T_{1}$ (ms) & $989 \pm 46^{\dagger}$ & $1083 \pm 59$ & $1118 \pm 51$ & 0.136 \\
\hline $\mathrm{T}_{2}$ (ms) & $54.1 \pm 2.0^{\dagger}$ & $57.0 \pm 3.2$ & $58.8 \pm 3.5$ & 0.242 \\
\hline
\end{tabular}

$\mathrm{p}$ values refer to the significance of the difference between no rejection and rejection. The significance of the difference between healthy volunteers and no rejection is denoted by ${ }^{*}(\mathrm{p}<0.05)$ or ${ }^{\dagger}(\mathrm{p}<0.01)$. LV indicates left ventricle; EDV end diastolic volume; ESV end-systolic volume; SV stroke volume; EF ejection fraction, $\varepsilon \subset c$ peak systolic circumferential strain; SR strain rate; \%AVC refers to time between peak electrocardiogram R wave and aortic valve closure expressed as a percentage. The suffix I indicates indexed to body surface area.

intermediate to values for non-significant and significant ACAR.

\section{Time from transplantation}

CMR findings according to scan number (i.e. time from transplantation) are displayed in Table 3. LV mass, $\varepsilon \mathrm{cC}$, native $T_{1}$ and $T_{2}$ decreased significantly, and MPR increased significantly, between scans 1 and 4 (Figure 4). Seven patients (32\%) demonstrated a pericardial effusion on scan 1, which had resolved in all but 2 patients by scan 2; pericardial effusion was not observed on scans 3 and 4 in any patient $(\mathrm{p}=0.002)$. On LGE imaging, one patient displayed evidence of an anterior myocardial infarction and another patient demonstrated right ventricular insertion point enhancement, but no temporal changes were observed. At the time of scan 4, LV volumetrics, EF, SR and $\mathrm{T}_{2}$ were not significantly different between transplant recipients and healthy volunteers, although other parameters remained abnormal.

\section{Influence of other factors on CMR findings}

Three patients (14\%) developed primary graft dysfunction (PGD). In these patients, mean baseline EF was $26.2 \pm$ $4.9 \%$ (versus $63.0 \pm 7.5 \%$ in patients without graft dysfunction, $\mathrm{p}<0.001$ ) and remained severely impaired across all 4 scans, albeit with some improvement (Table 4). Measures of contractile function and tissue characterization parameters were also markedly abnormal in these patients and were significantly different to patients without PGD (Table 4). In light of these findings, analysis of CMR data with regards to ACAR was repeated after adjusting for PGD, with results as follows (non-significant
ACAR vs. significant ACAR): $\varepsilon$ CC $14.4 \pm 3.2 \%$ vs. $12.7 \pm$ $2.5 \%, \mathrm{p}=0.015$; native $\mathrm{T}_{1} 1067 \pm 43 \mathrm{~ms}$ vs. $1118 \pm 51 \mathrm{~ms}$, $\mathrm{p}=0.100 ; \mathrm{T}_{2} 56.3 \pm 2.7 \mathrm{~ms}$ vs. $58.8 \pm 3.5 \mathrm{~ms}, \mathrm{p}=0.110$ (Figure 2).

Eight patients (36\%) were treated for CMV infection while taking part in the study (20 biopsies performed during CMV treatment). After adjusting for PGD, no CMR parameter differed significantly according to CMV infection.

\section{Relationship between CMR parameters}

There were significant linear relationships between LVEF and $\varepsilon \mathrm{cc}(\mathrm{LVEF}=-0.49 \varepsilon \mathrm{cc}+53 ; \mathrm{p}=0.044), \quad$ LVEF and peak systolic SR $(\mathrm{LVEF}=-8.9 \mathrm{SR}+51 ; \mathrm{p}=0.016)$, LVEF and $\mathrm{T}_{1}\left(\mathrm{LVEF}=-0.04 \mathrm{~T}_{1}+106 ; \mathrm{p}=0.006\right)$, LVEF and $\mathrm{T}_{2}$ $\left(\right.$ LVEF $\left.=-0.65 \mathrm{~T}_{1}+97 ; \mathrm{p}=0.005\right), \mathrm{T}_{1}$ and $\mathrm{T}_{2}\left(\mathrm{~T}_{1}=7.9 \mathrm{~T}_{2}+\right.$ 629; $\mathrm{p}<0.001) ; \mathrm{T}_{1}$ and $\varepsilon \mathrm{Cc}\left(\mathrm{T}_{1}=4.5 \varepsilon \mathrm{cc}+1148 ; \mathrm{p}=0.004\right)$; $\mathrm{T}_{2}$ and $\varepsilon \mathrm{CC}\left(\mathrm{T}_{2}=0.43 \varepsilon \mathrm{cc}+63 ; \mathrm{p}<0.001\right)$ and $\mathrm{T}_{2}$ and peak systolic $\mathrm{SR}\left(\mathrm{T}_{2}=4.2 \mathrm{SR}+61 ; \mathrm{p}=0.002\right)$.

\section{Discussion}

In this study CMR was not able to accurately detect ACAR in the early phase post-transplantation. However, this study does demonstrate the complexity of factors affecting allograft structure and function in this period and provides novel insight into the myocardial injury associated with transplantation, and its recovery.

Most published studies investigating the role of noninvasive ACAR surveillance techniques select patients known/suspected to have ACAR, and commonly include patients outside the time period when the early detection of ACAR is likely to be most useful [13]. In contrast, the 

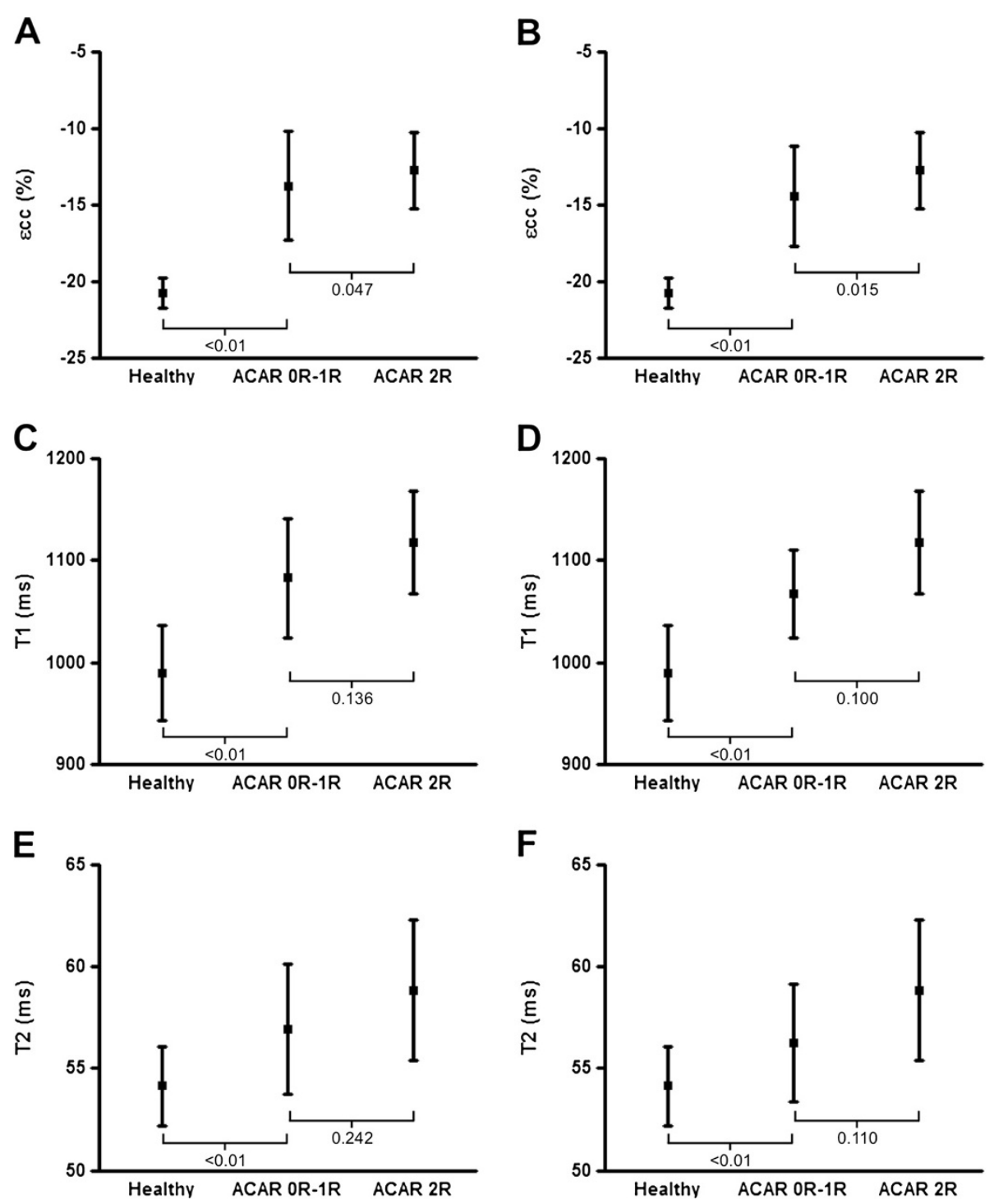

Figure 2 CMR parameters in significant and non-significant rejection and healthy volunteers. Peak systolic circumferential strain $(\varepsilon \subset C, A)$, myocardial $T_{1}$ relaxation time $\mathbf{( C )}$ and myocardial $T_{2}$ relaxation time $\mathbf{( E )}$ in significant (grade $2 \mathrm{R}$ ) and non-significant (grades $0 \mathrm{R}-1 \mathrm{R}$ ) acute cardiac allograft rejection (ACAR) and in matched healthy volunteers. B, D and $\mathbf{F}$ display corresponding data after patients with primary graft dysfunction have been excluded.

current study used an unselected cohort and focused on the time period when ACAR is of greatest clinical importance (i.e. the first 6 post-operative months) [1].

The per-patient incidence of significant ACAR in the current study (23\%) is in keeping with that reported in the most recent data from the ISHLT Registry [1]. Nevertheless, in keeping with other contemporary studies in this field, the number of episodes of significant ACAR captured (9\%) is relatively small and reflects the decreasing incidence of ACAR secondary to advances in immunosuppression [14]. Indeed, while the decreasing incidence of ACAR makes biopsy increasingly unattractive (the yield of biopsy is now of the same order of magnitude as its complication rate), the decreasing incidence makes the assessment of new surveillance techniques more difficult [2].

In the present study $\varepsilon \mathrm{cc}$ was significantly lower in grade $2 \mathrm{R}$ ACAR compared to grades $0 \mathrm{R}$ and $1 \mathrm{R}$, although the absolute difference was small and there was considerable overlap between groups, indeed by considering all data points as independent (i.e. by not taking into account the repeated measurements within each patient) which allows receiver operating characteristic (ROC) curve analysis to be performed, sensitivity and specificity of $\varepsilon \mathrm{cc}$ for 


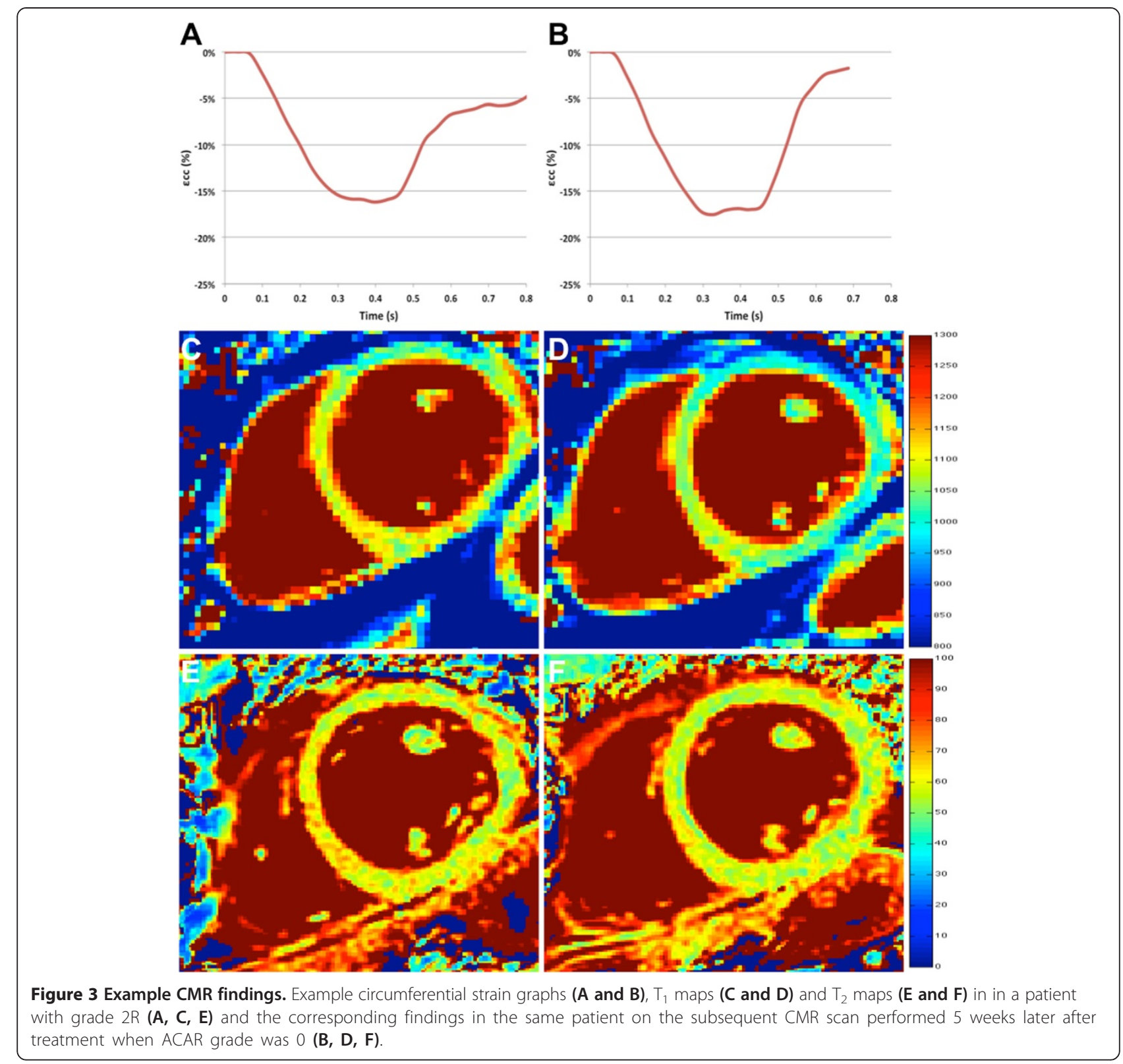

detecting significant ACAR were only $75 \%$ and $64 \%$ respectively (area under curve (AUC) 0.69, 95\% confidence intervals 0.53-0.85). In a rodent transplant model, Wu et al. [15] found regional impairment of $\varepsilon c c$, as assessed with CMR tagging, to correspond to areas of macrophage infiltration, however echocardiographic data in humans regarding the utility of strain (in all orthogonal directions) for detecting ACAR is inconsistent $[16,17]$.

Myocardial $T_{1}$ and $T_{2}$ relaxation times are sensitive to changes in myocardial water content and have been proposed to detect myocardial oedema in other conditions [18-20]. However, in the current study, myocardial $T_{1}$ and $\mathrm{T}_{2}$ were not significantly higher in grade $2 \mathrm{R}$ ACAR compared to grades $0 \mathrm{R}-1 \mathrm{R}$, although both demonstrated trends towards higher values, particularly after accounting for PGD.

In a recent study by Usman et al. [14], ACAR was associated with elevated myocardial $\mathrm{T}_{2}$, which is in keeping with the findings of an early CMR study by Marie et al. [21]. However, there are important differences between these studies and the current study. In the studies by Usman et al. and Marie et al. patients were substantially longer post-transplant than in the current study, thus reducing the effect of transplant-related myocardial injury, described below, on $\mathrm{T}_{2}$ measurements, but also missing the window in which early detection of ACAR is 
Table 3 CMR findings displayed according to scan number

\begin{tabular}{|c|c|c|c|c|c|c|}
\hline & $\begin{array}{l}\text { Scan } 1 \\
\text { (6.9 weeks) }\end{array}$ & $\begin{array}{l}\text { Scan } 2 \\
\text { (10.9 weeks) }\end{array}$ & $\begin{array}{l}\text { Scan } 3 \\
\text { (16.6 weeks) }\end{array}$ & $\begin{array}{l}\text { Scan } 4 \\
\text { (22.3 weeks) }\end{array}$ & $p$ value & $\begin{array}{l}\text { Healthy } \\
\text { volunteers }\end{array}$ \\
\hline LVEDVI $\left(\mathrm{mL} / \mathrm{m}^{2}\right)$ & $83.2 \pm 20.3$ & $83.4 \pm 23.2$ & $83.7 \pm 22.6$ & $82.6 \pm 19.9$ & 0.979 & $85.9 \pm 8.1$ \\
\hline LVESVI $\left(\mathrm{mL} / \mathrm{m}^{2}\right)$ & $35.3 \pm 21.1$ & $35.8 \pm 23.6$ & $34.5 \pm 21.2$ & $33.5 \pm 19.4$ & 0.423 & $28.5 \pm 4.3$ \\
\hline LVEF (\%) & $59.5 \pm 13.2$ & $60.1 \pm 14.2$ & $61.1 \pm 13.9$ & $61.3 \pm 12.2$ & 0.216 & $66.9 \pm 4.2$ \\
\hline LVMI $\left(\mathrm{g} / \mathrm{m}^{2}\right)$ & $63.6 \pm 15.7$ & $62.5 \pm 12.9$ & $62.1 \pm 12.8$ & $59.3 \pm 11.9$ & 0.001 & $46.4 \pm 7.3^{\dagger}$ \\
\hline$\varepsilon \subset c(\%)$ & $-12.4 \pm 3.8$ & $-13.5 \pm 2.9$ & $-14.2 \pm 3.8$ & $-14.4 \pm 3.0$ & 0.024 & $-20.7 \pm 1.0^{\dagger}$ \\
\hline Peak systolic SR (1/s) & $-1.05 \pm 0.33$ & $-1.03 \pm 0.27$ & $-1.07 \pm 0.33$ & $-1.05 \pm 0.30$ & 0.669 & $-1.17 \pm 0.14$ \\
\hline Early diastolic SR (1/s) & $0.29 \pm 0.13$ & $0.25 \pm 0.10$ & $0.23 \pm 0.12$ & $0.25 \pm 0.15$ & 0.499 & $0.37 \pm 0.20$ \\
\hline Normalised twist $(\% / m m)$ & $0.26 \pm 0.10$ & $0.25 \pm 0.10$ & $0.27 \pm 0.11$ & $0.27 \pm 0.08$ & 0.709 & $0.34 \pm 0.12^{*}$ \\
\hline Torsion (mm) & $7.68 \pm 2.89$ & $7.16 \pm 2.73$ & $7.78 \pm 3.19$ & $7.59 \pm 2.24$ & 0.761 & $8.99 \pm 3.11$ \\
\hline Time to peak torsion (\% AVC) & $101.0 \pm 11.0$ & $97.1 \pm 7.2$ & $97.9 \pm 6.3$ & $96.5 \pm 9.3$ & 0.466 & $96.4 \pm 5.5$ \\
\hline Native $T_{1}$ (ms) & $1109 \pm 53$ & $1089 \pm 62$ & $1084 \pm 70$ & $1063 \pm 40$ & $<0.001$ & $989 \pm 46^{\dagger}$ \\
\hline$T_{2}(m s)$ & $58.7 \pm 3.4$ & $57.1 \pm 3.4$ & $56.9 \pm 2.9$ & $55.9 \pm 2.9$ & 0.003 & $54.1 \pm 2.0$ \\
\hline Resting MBF $(\mathrm{mL} / \mathrm{min} / \mathrm{g})$ & - & $0.85 \pm 0.12$ & - & $0.86 \pm 0.16$ & 0.150 & $0.74 \pm 0.10$ \\
\hline Stress MBF $(\mathrm{mL} / \mathrm{min} / \mathrm{g})$ & - & $1.21 \pm 0.18$ & - & $1.37 \pm 0.26$ & 0.172 & $1.81 \pm 0.29^{\dagger}$ \\
\hline MPR & - & $1.44 \pm 0.22$ & - & $1.62 \pm 0.35$ & 0.023 & $2.46 \pm 0.34^{\dagger}$ \\
\hline ECV & - & $30.1 \pm 0.5$ & - & $28.0 \pm 1.6$ & $<0.001$ & $25.3 \pm 0.18^{\dagger}$ \\
\hline
\end{tabular}

Time between transplantation and scan is given in brackets.

$p$ values refer to the significance of the difference between scans. The significance of the difference between healthy volunteers and scan 4 is denoted by * $p<0.05)$ and $^{\dagger}(\mathrm{p}<0.01)$. MBF indicates myocardial blood flow; MPR myocardial perfusion reserve; LGE late gadolinium enhancement; ECV myocardial extracellular volume. Other abbreviations as per Table 2 .
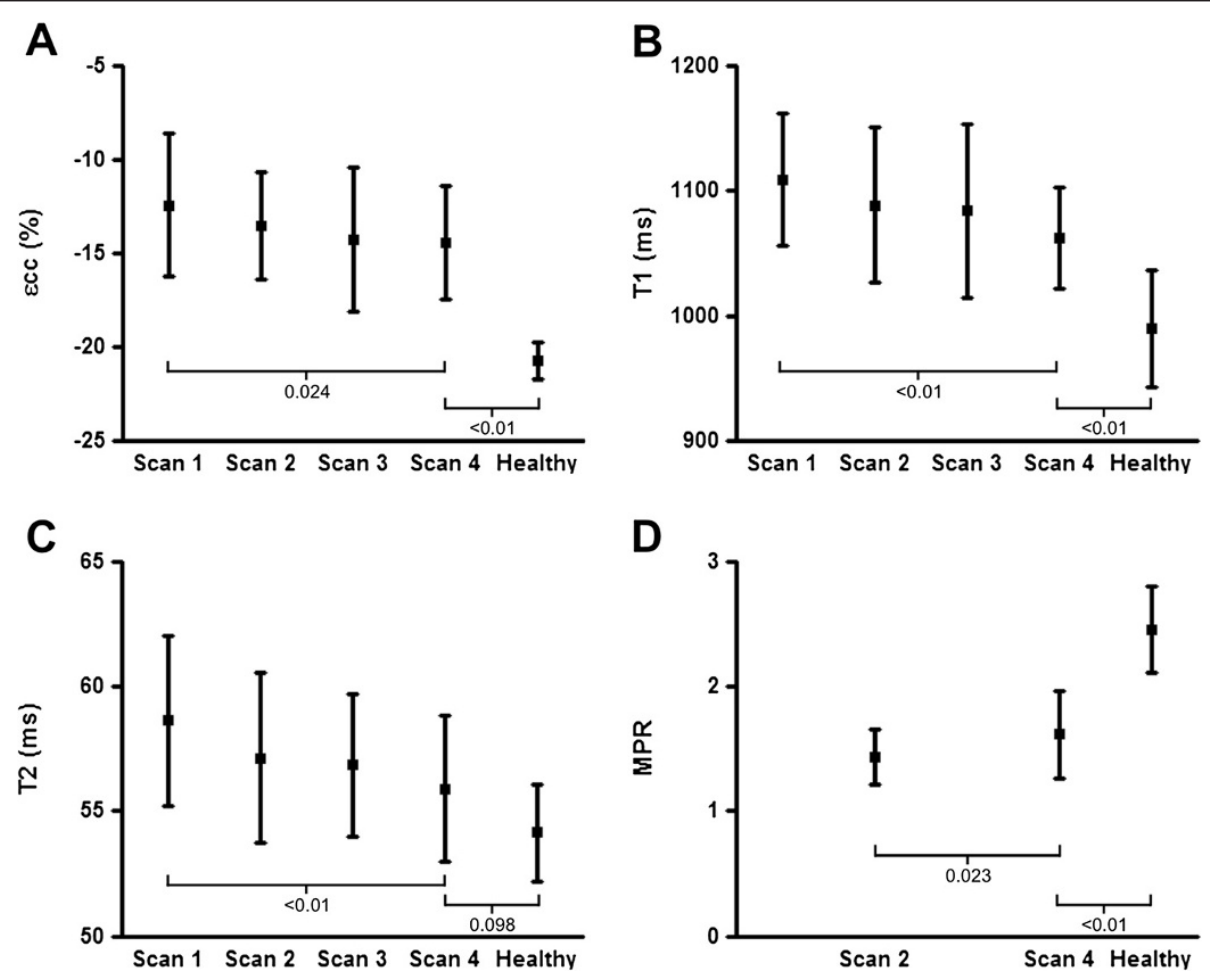

Figure 4 Change in CMR parameters over time from transplantation. Change in peak systolic circumferential strain ( $(\varepsilon \subset c, A)$, myocardial $T_{1}$ relaxation time (B), myocardial $T_{2}$ relaxation time $\mathbf{( C )}$ and myocardial perfusion reserve (MPR, D) over time from transplantation (scan 1: 6.9 weeks post-transplantation; scan 2: 10.9 weeks; scan 3: 16.6 weeks; scan 4: 22.3 weeks) and comparison of parameters at the time of scan 4 with matched healthy volunteers. 
Table 4 Selected CMR parameters displayed according to the presence of primary graft dysfunction (PGD)

\begin{tabular}{|c|c|c|c|c|c|}
\hline & Scan 1 (6.9 weeks) & Scan 2 (10.9 weeks) & Scan 3 (16.6 weeks) & Scan 4 (22.3 weeks) & $\mathrm{p}$ value \\
\hline \multicolumn{6}{|c|}{ LVEDVI $\left(\mathrm{mL} / \mathrm{m}^{2}\right)$} \\
\hline No PGD & $78.5 \pm 14.6$ & $76.4 \pm 15.5$ & $77.4 \pm 16.5$ & $77.7 \pm 14.1$ & \\
\hline PGD & $127.5 \pm 16.6^{\dagger}$ & $127.3 \pm 12.1^{\dagger}$ & $123.7 \pm 10.4^{\dagger}$ & $123.6 \pm 12.8^{\dagger}$ & $<0.001$ \\
\hline \multicolumn{6}{|c|}{ LVESVI $\left(\mathrm{mL} / \mathrm{m}^{2}\right)$} \\
\hline No PGD & $29.1 \pm 8.3$ & $27.3 \pm 8.9$ & $27.0 \pm 9.5$ & $27.9 \pm 9.2$ & \\
\hline PGD & $94.2 \pm 7.5^{\dagger}$ & $89.4 \pm 11.1^{\dagger}$ & $81.7 \pm 5.8^{\dagger}$ & $81.4 \pm 17.9^{\dagger}$ & $<0.001$ \\
\hline \multicolumn{6}{|l|}{ LVEF (\%) } \\
\hline No PGD & $63.0 \pm 7.5$ & $64.8 \pm 7.7$ & $65.4 \pm 8.7$ & $64.5 \pm 7.9$ & \\
\hline PGD & $26.2 \pm 4.9^{\dagger}$ & $29.9 \pm 2.2^{\dagger}$ & $33.7 \pm 5.8^{\dagger}$ & $34.5 \pm 7.8^{\dagger}$ & $<0.001$ \\
\hline \multicolumn{6}{|l|}{$\varepsilon \subset c(\%)$} \\
\hline No PGD & $-13.1 \pm 3.6$ & $-14.0 \pm 2.6$ & $-15.0 \pm 3.5$ & $-14.9 \pm 2.9$ & \\
\hline PGD & $-8.5 \pm 3.3^{*}$ & $-9.3 \pm 1.7^{*}$ & $-9.3 \pm 1.6^{*}$ & $-10.9 \pm 0.6$ & $<0.001$ \\
\hline \multicolumn{6}{|c|}{ Native $T_{1}$ (ms) } \\
\hline No PGD & $1099 \pm 42$ & $1067 \pm 42$ & $1066 \pm 59$ & $1055 \pm 32$ & \\
\hline$P G D$ & $1167 \pm 79^{*}$ & $1184 \pm 66^{\dagger}$ & $1177 \pm 50^{+}$ & $1122 \pm 66^{*}$ & 0.001 \\
\hline \multicolumn{6}{|l|}{$\mathrm{T}_{2}(\mathrm{~ms})$} \\
\hline No PGD & $58.0 \pm 2.88$ & $56.6 \pm 3.2$ & $56.2 \pm 2.7$ & $55.3 \pm 2.3$ & \\
\hline PGD & $64.7 \pm 0.93^{\dagger}$ & $60.5 \pm 3.4$ & $60.3 \pm 0.5^{*}$ & $60.8 \pm 3.0^{+}$ & $<0.001$ \\
\hline
\end{tabular}

thought to be most useful (also see below). In addition, both studies specifically selected patients known/suspected of having ACAR and different definitions of 'significant' ACAR were used. Finally, neither study made statistical adjustment for repeated measurements within the same patients (for example 33 patients in the study by Marie et al. underwent $2-4$ CMR scans). Indeed if equivalent statistical methods to those used by Usman and Marie are applied here, the differences in $T_{2}$ (and $T_{1}$ ) between ACAR groups after accounting for PGD become significant $\left(\mathrm{T}_{2}: \mathrm{p}=0.016 ; \mathrm{T}_{1}: \mathrm{p}=0.008\right)$ although the sensitivity and specificity remain modest $\left(\mathrm{T}_{2}: 75 \%\right.$ and $67 \%$ respectively, AUC 0.74 (0.53-0.95); $\mathrm{T}_{1}: 83 \%$ and $72 \%$ respectively, AUC 0.79 (0.59-0.98)). The role of myocardial $\mathrm{T}_{1}$ in ACAR has not been previously evaluated using contemporary CMR techniques.

Whilst recognizing that CMR methods requiring gadolinium contrast agent would be undesirable for ACAR surveillance, post-contrast CMR techniques were included in order to provide further characterization of ACAR pathophysiology. However the size of the cohort studied and prevalence of renal impairment, typical of many studies involving transplant patients, meant insufficient patients with ACAR underwent contrast-enhanced CMR to allow meaningful comparison of these parameters.

This study does serve to provide detailed characterization of the evolution of LV structure and function during the early phase post-transplantation. Over the first 5 postoperative months significant improvements were seen in markers of LV structure (mass) and contractility ( $\varepsilon \mathrm{cc})$, proposed markers of myocardial oedema (native $\mathrm{T}_{1}, \mathrm{~T}_{2}$ and ECV) and microvascular function (MPR), although few parameters normalized.

The insults to which the donor heart is subjected in the peri-transplant period, including brain death and its sequelae, ischemia and reperfusion, are likely to cause considerable myocardial injury, despite the preservation of gross markers of cardiac function (e.g. EF) in most patients. The current study comprehensively characterizes this myocardial injury for the first time, suggesting that it manifests as myocardial oedema, microvascular dysfunction and, likely as a consequence of both of these factors and of direct myocyte injury, impaired contractile function. The study also provides insight into its natural history, demonstrating how the injury improves over the first 5-months post-transplant but also showing that it persists for at least this period, with few parameters returning to normal over this time. Furthermore it is of considerable interest to note that proposed markers of oedema were significantly higher in patients that developed PGD compared to other allograft recipients, and remained elevated over the period studied. The mechanisms of myocardial stunning, seen in ischemic heart disease, include myocardial oedema; possibly via increasing 
the distance between actin and myosin filaments, which in turn leads to reduced contractility [22,23]. The pathophysiological mechanisms of PGD are not well understood and are likely multifactorial, but the results of this study suggest that, analogous to myocardial stunning, oedema may play an important role.

There has been little previous investigation into temporal changes in allograft structure and function in the early phase post-transplant. Using echocardiography, Antunes et al. [24] found LV EF to improve significantly over the first month post-transplantation. Eleid et al. [17] found $\varepsilon c c$, assessed using serial speckle-tracking echocardiography, remained markedly impaired compared to healthy subjects throughout the first two post-operative years although degree of temporal change was not reported. Wisenburg et al. [25] using spin echo sequences at $0.15 \mathrm{~T}$, found myocardial $T_{1}$ and $T_{2}$ to be elevated in all patients during the very early period post-transplant however values returned to normal 25 days post-transplant. In the current study myocardial $\mathrm{T}_{1}$ and $\mathrm{T}_{2}$ remained elevated for considerably longer, which is in keeping with contemporary CMR findings in other pathologies such as myocardial infarction or myocarditis [23]. Finally in keeping with the current study, Preumont et al. [26] demonstrated higher MPR, as assessed using Nitrogen-13 PET, in patients with angiographically normal epicardial coronary arteries scanned at 9-months post-transplant compared to matched patients scanned at 3-months post-transplant.

Taking the findings of the current study and of Usman et al. [14] together it may be that CMR parameters become more useful for detecting ACAR as time from transplantation increases and the transplant-related myocardial injury subsides. The paradox however is that while noninvasive approaches to ACAR surveillance may become more discriminatory as time from transplantation increases, the benefit of the early detection of ACAR diminishes, indeed the usefulness of routine screening later than one year post-transplant is subject to debate $[2,27]$.

\section{Limitations}

Despite over 2 years of recruitment and a recruitment rate of over $75 \%$ in those eligible, the number of patients included is relatively small. This is in part reflective of the robust study design, although the size of the cohort and number of scans performed here are in keeping with many studies assessing non-invasive approaches to ACAR surveillance. As acknowledged earlier, the number of episodes of significant ACAR captured is also relatively small. It is also recognized that biopsy is limited as a reference standard, with 'biopsy-negative' ACAR widely reported [3], however patients were followed-up in order to identify those treated for ACAR in the absence of positive biopsy. Baseline post-transplant coronary angiography was not performed and as such epicardial coronary disease cannot be excluded as a cause of the low MPR seen in transplant recipients, however given that MPR improved significantly over time this is unlikely. Finally, as is well documented elsewhere, histological validation of T1 and T2 imaging for detecting and quantifying non-infarct related myocardial oedema is lacking. Nevertheless, our application of T1 and T2 sequences is in keeping with contemporary literature.

\section{Conclusions}

In this study, multiparametric CMR demonstrated that multiple factors affect cardiac allograft structure and function in the early phase post-transplantation. Whilst CMR provided novel insight into the myocardial injury associated with transplantation, it was not able to accurately detect ACAR as diagnosed by biopsy during this period.

\section{Abbreviations}

ACAR: Acute cardiac allograft rejection; CMR: Cardiovascular magnetic resonance; ECV: Myocardial extracellular volume; EF: Ejection fraction; LGE: Late gadolinium enhancement; LV: Left ventricle; MBF: Myocardial blood flow; MPR: Myocardial perfusion reserve; PGD: Primary graft dysfunction: ROC: Receiver operating characteristic.

\section{Competing interests}

The authors declare that they have no competing interest.

\section{Authors' contributions}

All authors have contributed significantly to the work. CAM and MS conceived and directed the project. JHN and CAM developed the CMR analysis tools with input from GC. CAM, SMS, NY and SGW recruited the patients. DC, CAM, MPA and MS performed the CMR scanning and analysis. PB performed the histological analysis. All authors provided critical review of the manuscript. All authors read and approved the final manuscript.

\section{Acknowledgements}

CAM is supported by a Fellowship from the National Institute for Health Research, UK and received research funding from the Mason Medical Foundation. CAM, SGW, NY and MS have received research funding from New Start Transplant Charity. The authors would like to thank the radiographers and administrative staff of the Alliance Wythenshawe CMR Unit, the secretarial and nursing staff of The Transplant Unit, Wythenshawe, UK, and the patients of The Transplant Unit whose willing cooperation made this work possible.

\section{Author details}

${ }^{1}$ North West Heart Centre and The Transplant Centre, University Hospital of South Manchester, Manchester, UK. ${ }^{2}$ Centre for Imaging Sciences \& Biomedical Imaging Institute, University of Manchester, Manchester, UK. ${ }^{3}$ Institute of Cardiovascular Sciences, University of Manchester, Manchester, UK. ${ }^{4}$ Alliance Medical Cardiac MRI Unit, Wythenshawe Hospital, Manchester, UK. ${ }^{5}$ Department of Pathology, University Hospital of South, Manchester, UK. ${ }^{6}$ Christie Medical Physics and Engineering, The Christie Hospital, Manchester, UK.

Received: 1 April 2014 Accepted: 30 June 2014

Published: 20 July 2014

\section{References}

1. Stehlik J, Edwards LB, Kucheryavaya AY, Benden C, Christie JD, Dipchand Al, Dobbels F, Kirk R, Rahmel AO, Hertz MI. The registry of the International Society for Heart and Lung Transplantation: 29th official adult heart transplant report-2012. J Heart Lung Transplant. 2012; 31:1052-64. 
2. Hamour IM, Burke MM, Bell AD, Panicker MG, Banerjee R, Banner NR. Limited utility of endomyocardial biopsy in the first year after heart transplantation. Transplantation. 2008; 85:969-74.

3. Subherwal S, Kobashigawa JA, Cogert G, Patel J, Espejo M, Oeser B. Incidence of acute cellular rejection and non-cellular rejection in cardiac transplantation. Transplant Proc. 2004; 36:3171-2.

4. Messroghli DR, Greiser A, Frohlich M, Dietz R, Schulz-Menger J. Optimization and validation of a fully-integrated pulse sequence for modified look-locker inversion-recovery (MOLLI) T1 mapping of the heart. J Magn Reson Imaging. 2007; 26:1081-6.

5. Kellman P, Aletras AH, Mancini C, McVeigh ER, Arai AE. T2-prepared SSFP improves diagnostic confidence in edema imaging in acute myocardial infarction compared to turbo spin echo. Magn Reson Med. 2007; 57:891-7.

6. Miller CA, Naish JH, Ainslie MP, Tonge C, Tout D, Arumugam P, Banerji A, Egdell RM, Clark D, Weale P, Steadman CD, McCann GP, Ray SG, Parker GJ, Schmitt M. Voxel-wise quantification of myocardial blood flow with cardiovascular magnetic resonance: effect of variations in methodology and validation with positron emission tomography. J Cardiovasc Magn Reson. 2014; 16:11

7. Miller CA, Jordan P, Borg A, Argyle R, Clark D, Pearce K, Schmitt M. Quantification of left ventricular indices from SSFP cine imaging: Impact of real-world variability in analysis methodology and utility of geometric modeling. J Magn Reson Imaging. 2013; 37:1213-22.

8. Miller CA, Borg A, Clark D, Steadman CD, McCann GP, Clarysse P, Croisille P, Schmitt M. Comparison of local sine wave modeling with harmonic phase analysis for the assessment of myocardial strain. J Magn Reson Imaging. 2013; 38:320-8.

9. Miller CA, Naish JH, Bishop P, Coutts G, Clark D, Zhao S, Ray SG, Yonan N Williams SG, Flett AS, Moon JC, Greiser A, Parker GJ, Schmitt M. Comprehensive validation of cardiovascular magnetic resonance techniques for the assessment of myocardial extracellular volume. Circ Cardiovasc Imaging. 2013; 6:373-83.

10. Biglands J, Magee D, Boyle R, Larghat A, Plein S, Radjenovic A. Evaluation of the effect of myocardial segmentation errors on myocardial blood flow estimates from DCE-MRI. Phys Med Biol. 2011; 56:2423-43.

11. Jerosch-Herold M, Swingen C, Seethamraju RT. Myocardial blood flow quantification with MRI by model-independent deconvolution. Med Phys. 2002; 29:886-97.

12. Stewart S, Winters GL, Fishbein MC, Tazelaar HD, Kobashigawa J, Abrams J, Andersen CB, Angelini A, Berry GJ, Burke MM, Demetris AJ, Hammond E, Itescu S, Marboe CC, McManus B, Reed EF, Reinsmoen NL, Rodriguez ER, Rose AG, Rose M, Suciu-Focia N, Zeevi A, Billingham ME. Revision of the 1990 working formulation for the standardization of nomenclature in the diagnosis of heart rejection. J Heart Lung Transplant. 2005; 24:1710-20.

13. Miller CA, Fildes JE, Ray SG, Doran H, Yonan N, Williams SG, Schmitt M. Non-invasive approaches for the diagnosis of acute cardiac allograft rejection. Heart. 2013; 99:445-53.

14. Usman AA, Taimen K, Wasielewski M, McDonald J, Shah S, Giri S, Cotts W, McGee E, Gordon R, Collins JD, Markl M, Carr JC. Cardiac Magnetic Resonance T2 Mapping in the Monitoring and Follow-up of Acute Cardiac Transplant Rejection: A Pilot Study. Circ Cardiovasc Imaging. 2012; 5:782-90.

15. Wu YL, Ye Q, Sato K, Foley LM, Hitchens TK, Ho C. Noninvasive evaluation of cardiac allograft rejection by cellular and functional cardiac magnetic resonance. JACC CardiovasC Imaging. 2009; 2:731-41.

16. Roshanali F, Mandegar MH, Bagheri J, Sarzaeem MR, Chitsaz S, Alaeddini F, Saidi B. Echo rejection score: new echocardiographic approach to diagnosis of heart transplant rejection. Eur J Cardiothorac Surg. 2010; 38:176-80.

17. Eleid MF, Caracciolo G, Cho EJ, Scott RL, Steidley DE, Wilansky S, Arabia FA, Khandheria BK, Sengupta PP. Natural history of left ventricular mechanics in transplanted hearts: relationships with clinical variables and genetic expression profiles of allograft rejection. JACC Cardiovasc Imaging. 2010; 3:989-1000.

18. Higgins CB, Herfkens R, Lipton MJ, Sievers R, Sheldon P, Kaufman L, Crooks $L E$. Nuclear magnetic resonance imaging of acute myocardial infarction in dogs: alterations in magnetic relaxation times. Am J Cardiol. 1983; 52:184-8.

19. Ugander M, Bagi PS, Oki AJ, Chen B, Hsu LY, Aletras AH, Shah S, Greiser A, Kellman P, Arai AE. Myocardial edema as detected by pre-contrast T1 and T2 CMR delineates area at risk associated with acute myocardial infarction. JACC CardiovasC Imaging. 2012; 5:596-603.
20. Thavendiranathan P, Walls M, Giri S, Verhaert D, Rajagopalan S, Moore S, Simonetti OP, Raman SV. Improved detection of myocardial involvement in acute inflammatory cardiomyopathies using T2 mapping. Circ CardiovasC Imaging. 2012; 5:102-10.

21. Marie PY, Angioi M, Carteaux JP, Escanye JM, Mattei S, Tzvetanov K, Claudon O, Hassan N, Danchin N, Karcher G, Bertrand A, Walker PM, Villemot JP. Detection and prediction of acute heart transplant rejection with the myocardial $\mathrm{T} 2$ determination provided by a black-blood magnetic resonance imaging sequence. J Am Coll Cardiol. 2001; 37:825-31.

22. Bragadeesh $T$, Jayaweera AR, Pascotto M, Micari A, Le DE, Kramer CM, Epstein FH, Kaul S. Post-ischaemic myocardial dysfunction (stunning) results from myofibrillar oedema. Heart. 2008; 94:166-71.

23. Kidambi A, Mather AN, Swoboda P, Motwani M, Fairbairn TA, Greenwood $J P$, Plein S. Relationship between Myocardial Edema and Regional Myocardial Function after Reperfused Acute Myocardial Infarction: An MR Imaging Study. Radiology. 2013; 267:701-8.

24. Antunes ML, Spotnitz HM, Clark MB, Steinhardt MJ, Marboe CC, Smith CR, Rose EA, Reemtsma K. Long-term function of human cardiac allografts assessed by two-dimensional echocardiography. J Thorac Cardiovasc Surg. 1989; 98:275-84

25. Wisenberg G, Pflugfelder PW, Kostuk WJ, McKenzie FN, Prato FS. Diagnostic applicability of magnetic resonance imaging in assessing human cardiac allograft rejection. Am J Cardiol. 1987; 60:130-6.

26. Preumont N, Berkenboom G, Vachiery J, Jansens J, Antoine M, Wikler D, Damhaut P, Degre S, Lenaers A, Goldman S. Early alterations of myocardial blood flow reserve in heart transplant recipients with angiographically normal coronary arteries. J Heart Lung Transplant. 2000; 19:538-45.

27. White JA, Guiraudon C, Pflugfelder PW, Kostuk WJ. Routine surveillance myocardial biopsies are unnecessary beyond one year after heart transplantation. J Heart Lung Transplant. 1995; 14:1052-6.

doi:10.1186/s12968-014-0052-6

Cite this article as: Miller et al:: Multiparametric cardiovascular magnetic resonance surveillance of acute cardiac allograft rejection and characterisation of transplantation-associated myocardial injury: a pilot study. Journal of Cardiovascular Magnetic Resonance 2014 16:52

\section{Submit your next manuscript to BioMed Central and take full advantage of:}

- Convenient online submission

- Thorough peer review

- No space constraints or color figure charges

- Immediate publication on acceptance

- Inclusion in PubMed, CAS, Scopus and Google Scholar

- Research which is freely available for redistribution 